\title{
Status of the HERA Facility
}

Collisions between high-energy electrons and protons could be observed for the first time in HERA with the two detectors ZEUS and H1 in May 1992. This concluded an 8-year construction period which started with the authorization of the HERA project in 1984. Throughout 1992, the electron and proton currents in the two HERA rings remained far below their design values, and the accumulated luminosity for the year was about 60 inverse nanobarns at each of the two detector locations. The number of particle bunches in each beam was increased in 1993 from 9 to 90 (the design value is 210) and average currents during luminosity operation were $20 \mathrm{~mA}$ (design: $58 \mathrm{~mA}$ ) for electrons and $20 \mathrm{~mA}$ (design: 163 $\mathrm{mA}$ ) for protons. The maximum luminosity observed was $1.4 \times 10^{30} \mathrm{~cm}^{-2} \mathrm{~s}^{-1}$, almost $10 \%$ of the design level of $1.6 \times 10^{31} \mathrm{~cm}^{-2} \mathrm{~s}^{-1}$. The accumulated luminosity in 1993 exceeded $1000 \mathrm{nb}^{-1}$ for both detectors. In order to reach the full design luminosity, the number of bunches in each beam must now be increased from 90 to 210 and the charge per bunch must be brought up to the design values. Two problems have to be overcome, namely:

1) While it is not difficult to reach the required bunch charge in the electron beam, the average current for the electrons is limited. It seems that positively charged dust particles become trapped in the electron beam when the average current is relatively large, thus causing a sharp decrease in lifetime. This effect limited HERA's average electron current in 1993 to $20 \mathrm{~mA}$.

2) The required number of protons per bunch of $1.0 x$ $10^{11}$ could be reached in the $7.5 \mathrm{GeV}$ proton synchrotron DESY III, but longitudinal multibunch instabilities prevented this full current from being accepted and accelerated to $40 \mathrm{GeV}$ in the PETRA II accelerator that follows.

Both problems (dust trapping in the electron ring and the longitudinal multibunch instability in DESY III) are well understood and are being worked on. Recently, a multibunch feedback system was brought into operation in DESY II which successfully eliminated the longitudinal instability. Most important, it has been established that the electron-proton spacecharge effects which present the principal luminosity limitation are as assumed in the design, implying that the design goals should be attainable in the not-toodistant future.

\section{Spin Polarization}

The build-up of transverse spin polarization, induced by the emission of synchrotron radiation, has been observed in the electron ring. Through careful orbit correction and the application of special orbit bumps it has been possible to increase the polarization to nearly $70 \%$. In order to generate an electron beam of well-defined helicity, an electron polarization parallel to the direction of motion is needed. A magnetic arrangement to turn the vertical spin into the longitudinal direction (a pair of spin rotators) was therefore installed in one of the HERA interaction regions during the 1993/94 winter shutdown, and was recently brought into operation for the first time. A (longitudinal) electron-spin polarization of $55 \%$ was observed during the very first test (it has since been increased to $65 \%$ ). This is the first time that electrons with longitudinal polarization have been produced in a storage ring. The accompanying figure shows the build-up of spin polarization as measured by observing the vertical asymmetry of back-scattered, circularly polarized light.

\section{Performance of the Cryogenics}

The superconducting magnets and the cryogenic system are the most challenging components of the HERA ring. A total of 2156 superconducting magnets have to be supplied with helium at $4.35 \mathrm{~K}$. A central cryogenic plant consisting of 3 identical $6.6 \mathrm{~kW}$ refrigerators together with 3 parallel compressor and gas-cleaning systems supply supercritical helium at 2.4 bar and $4.35 \mathrm{~K}$ to each of the 8 octants of the HERA proton ring via a transfer line. After flowing through one octant and thereby cooling the superconducting coils in that octant, the helium is expanded in a Joule-Thompson valve and transformed into two-phase helium which then flows back through the octant, thereby cooling the single-phase supercritical helium in contact with the superconducting coils. Heat produced in the superconducting coils is thus absorbed by the evaporation process in the two-phase helium. After flowing back in each octant, the two-phase helium is separated into its liquid and gaseous components, the latter being returned through a transfer line at $4.3 \mathrm{~K}$ to the central refrigerators. The same multi-pipe transfer line, which supplies the magnets with the supercritical helium and takes back the cold gaseous helium, also provides $40 \mathrm{~K}$ gaseous helium for the various heat shields and returns gas at temperatures of up to $80 \mathrm{~K}$. Finally, the transfer line is used to bring liquid helium to the superconducting coils of the detector magnets. A separate pipe, at ambient temperature, is used to catch any gaseous helium in the event of a quench of a superconducting magnet. A large central storage battery of helium pressure tanks can store the whole $15000 \mathrm{~kg}$ helium inventory of the HERA ring.

The cool-down of the entire HERA ring takes about 140 hours, and is determined by the permissible mechanical stresses of the superconducting magnets. The heat load of the magnets alone is $3.6 \mathrm{~kW}$ at $4.4 \mathrm{~K}$ and $19.6 \mathrm{~kW}$ at the shield temperature, and the total heat load including transfer lines is $5.1 \mathrm{~kW}$ at $4.4 \mathrm{~K}$ and $28.5 \mathrm{~kW}$ at the shield level. In addition, $10 \mathrm{~g}$ of liquid helium per second are necessary to cool the input of the current leads for the superconducting magnets.

All the cryogenic needs taken together require a total power of $3 \mathrm{MW}$. This is a genuine bargain if one compares the value of $3 \mathrm{MW}$ with the power consumption of normally conducting proton synchrotrons (e.g., the $310 \mathrm{GeV}$ SPS at CERN needs $65 \mathrm{MW}$ for operation). The required cryogenic power, even including the 2 superconducting solenoids for the two detectors and the 8 cryostats for 16 four-cell superconducting accelerating cavities for the HERA electron ring, is so low that 2 of the 3 parallel cryogenic plants easily satisfy the cryogenic needs. The third cryo-plant can therefore always be in a stand-by position or be available for maintenance and repair work. Each of the 3 cryo-plants has now run for some 30000 hours with an availability of approximately $99 \%$ of the scheduled time. With the exception of one special reference magnet installed outside the HERA ring, no single superconducting magnet has had to be changed since the proton ring was commissioned 4 years ago.

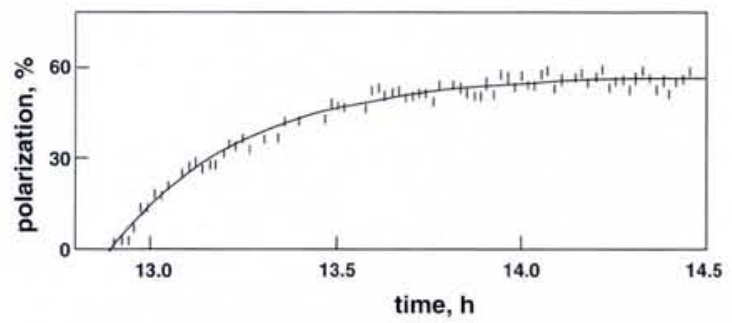

The build-up of radiation-induced electron spin polarization in HERA using activated spin rotators.
Looking along HERA's tunnel. The proton beam-line's string of liquidhelium cooled magnets in the cryogenic vacuum vessel lies above the electon beam-line's conventional electromagnets

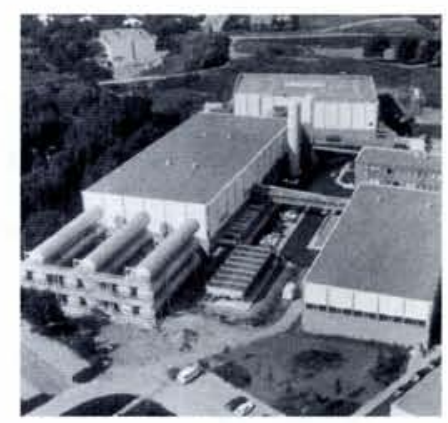

DESY's liquid helium storage plant is the largest in the world.

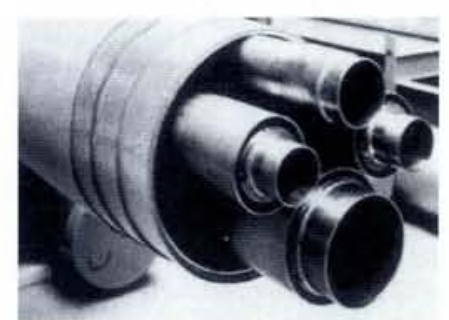

The heat shield and the croygen pipes that are located inside the vacuum vessel of HERA's proton ring. Liquid helium flows from the cryogen plant via the largest pipe and returns via one of the two, adajcent smaller pipes. The other adjacent pipe is for He gas at $40 \mathrm{~K}$ (to cool heat shields) which returns at tempe. ratures of up to $80 \mathrm{~K}$ via the upper. most pipe. 